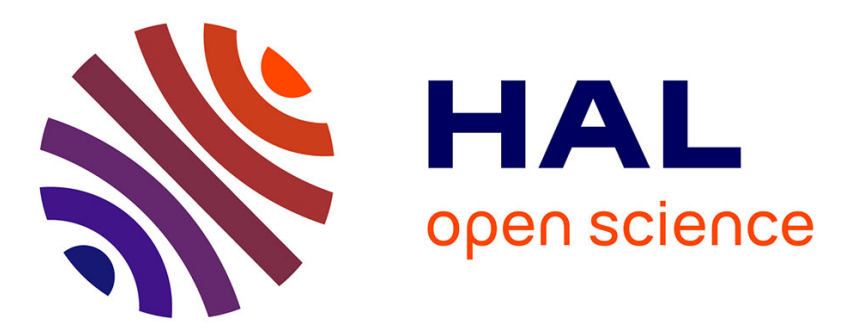

\title{
Étude de la lumière de fluorescence d'atomes excités en interaction résonnante avec un laser. II. Vérification expérimentale application au niveau 2p4 du néon
}

\author{
B. Decomps, M. Dumont
}

\section{- To cite this version:}

B. Decomps, M. Dumont. Étude de la lumière de fluorescence d'atomes excités en interaction résonnante avec un laser. II. Vérification expérimentale application au niveau $2 \mathrm{p} 4 \mathrm{du}$ néon. Journal de Physique, 1968, 29 (5-6), pp.443-455. 10.1051/jphys:01968002905-6044300 . jpa-00206670

HAL Id: jpa-00206670

https://hal.science/jpa-00206670

Submitted on 1 Jan 1968

HAL is a multi-disciplinary open access archive for the deposit and dissemination of scientific research documents, whether they are published or not. The documents may come from teaching and research institutions in France or abroad, or from public or private research centers.
L'archive ouverte pluridisciplinaire HAL, est destinée au dépôt et à la diffusion de documents scientifiques de niveau recherche, publiés ou non, émanant des établissements d'enseignement et de recherche français ou étrangers, des laboratoires publics ou privés. 


\title{
ÉTUDE DE LA LUMIÈRE DE FLUORESGENGE D'ATOMES EXGITÉS EN INTERAGTION RÉSONNANTE AVEG UN LASER II. VÉRIFIGATION EXPÉRIMENTALE
APPLIGATION AU NIVEAU $2 p_{4}$ DU NÉON (1)
}

\author{
Par B. DECOMPS et M. DUMONT, \\ Faculté des Sciences de Paris, \\ Laboratoire de Spectroscopie Hertzienne de l'E.N.S., associé au C.N.R.S.
}

(Reçu le 22 septembre 196\%.)

\begin{abstract}
Résumé. - La lumière de fluorescence émise par des atomes de néon d'une cellule $\mathrm{C}$ soumise à un champ magnétique et placée à l'intérieur de la cavité d'un laser à gaz est modifiée lors de l'irradiation par la raie laser $\lambda=6328 \AA\left(3 s_{2}-2 p_{4}\right)$ du néon.

L'étude détaillée de ces modifications sur les raies de fluorescence émises par le niveau $2 p_{4}$ permet de vérifier les prévisions théoriques développées dans un article antérieur (2). Elle montre, en particulier, l'importance de l'effet de cascade par émission spontanée sur la transition $\lambda=6328 \AA$. On trouve que les temps de relaxation de la population et de l'alignement du niveau $2 p_{4}$ sont très différents. Les sections efficaces de collision correspondantes ont été mesurées. De plus, on a mis en évidence un phénomène de diffusion multiple cohérente sur la raie $5943 \AA\left(2 p_{4}-1 s_{5}\right)$ sensible au courant de la décharge excitatrice de C.

Abstract. - The fluorescence light emitted by excited neon atoms of a cell $\mathrm{C}$, placed inside a laser cavity, is strongly changed when a 6328 A laser beam illuminates the cell. A magnetic field is applied on $\mathrm{C}$.

Detailed study of this effect on the fluorescence lines emitted by the $2 p_{4}$ level shows good agreement with a theoretical analysis published previously $\left(^{2}\right.$. It shows the importance of cascade effects due to spontaneous emission on the $3 s_{2}-2 p_{4}$ transition. Population and alignment relaxation times are found to be quite different. Corresponding collision cross-sections have been measured. The effect of "coherent" imprisonment of the $5943 \AA$ line $\left(2 p_{4}-1 s_{5}\right)$, sensitive to the discharge inside $C$, has been observed.
\end{abstract}

A. Le montage expérimental [1] à [4]. - L'ensemble des mesures repose sur l'analyse des variations des caractéristiques de la lumière de fluorescence émise par des atomes de néon excités par une décharge lorsqu'ils sont soumis à l'irradiation d'un laser de polarisation linéaire.

Pour cela, une cellule $\mathrm{G}$ remplie d'un mélange d'hélium et de néon en proportions variables est excitée par une décharge continue d'intensité réglable. $\mathrm{G}$ est placée à l'intérieur même de la cavité laser. En outre, un champ magnétique $H$, parallèle à l'axe, lui est appliqué. L'oscillation laser est assurée par un tube $\mathrm{L}$ beaucoup plus long que $\mathrm{C}$, dans lequel la nature du mélange hélium-néon et l'intensité de la décharge sont ajustées pour obtenir le maximum d'intensité lumineuse. Le tube $\mathrm{L}$ est amplificateur pour plusieurs raies du néon. Le choix de la longueur

(1) Cette étude a été réalisée avec l'aide de la D.R.M.E.

(2) Journal de Physique, 1968, 29, 181. Toutes les références, formules et paragraphes de cet article théorique sont précédés de I. d'onde d'oscillation est assuré par des miroirs à multicouches diélectriques dont le coefficient de réflexion n'est proche de 1 que pour une faible gamme de longueur d'onde ( fig. 1 a et $1 \mathrm{c}$ ).

Quel que soit le choix des miroirs, l'oscillation peut se produire sur la longueur d'onde :

$$
\lambda=3,39 \mu\left(3 s_{2}-3 p_{4}\right) .
$$

L'introduction de méthane dans les intervalles libres de la cavité permet d'absorber cette raie et de bloquer cette oscillation. Le laser oscille alors uniquement sur une des autres transitions possibles sur notre montage. Dans la quasi-totalité des expériences décrites dans cet article, nous avons utilisé l'oscillation laser sur la raie $\lambda=6328 \AA\left(3 s_{2}-2 p_{4}\right)$ qui nous a conduits à l'étude des niveaux $3 s_{2}$ et $2 p_{4}$.

On observe la lumière de fluorescence émise par $\mathrm{C}$ perpendiculairement à l'axe du laser simultanément dans deux directions respectivement parallèles et perpendiculaires à la direction de polarisation du laser. 


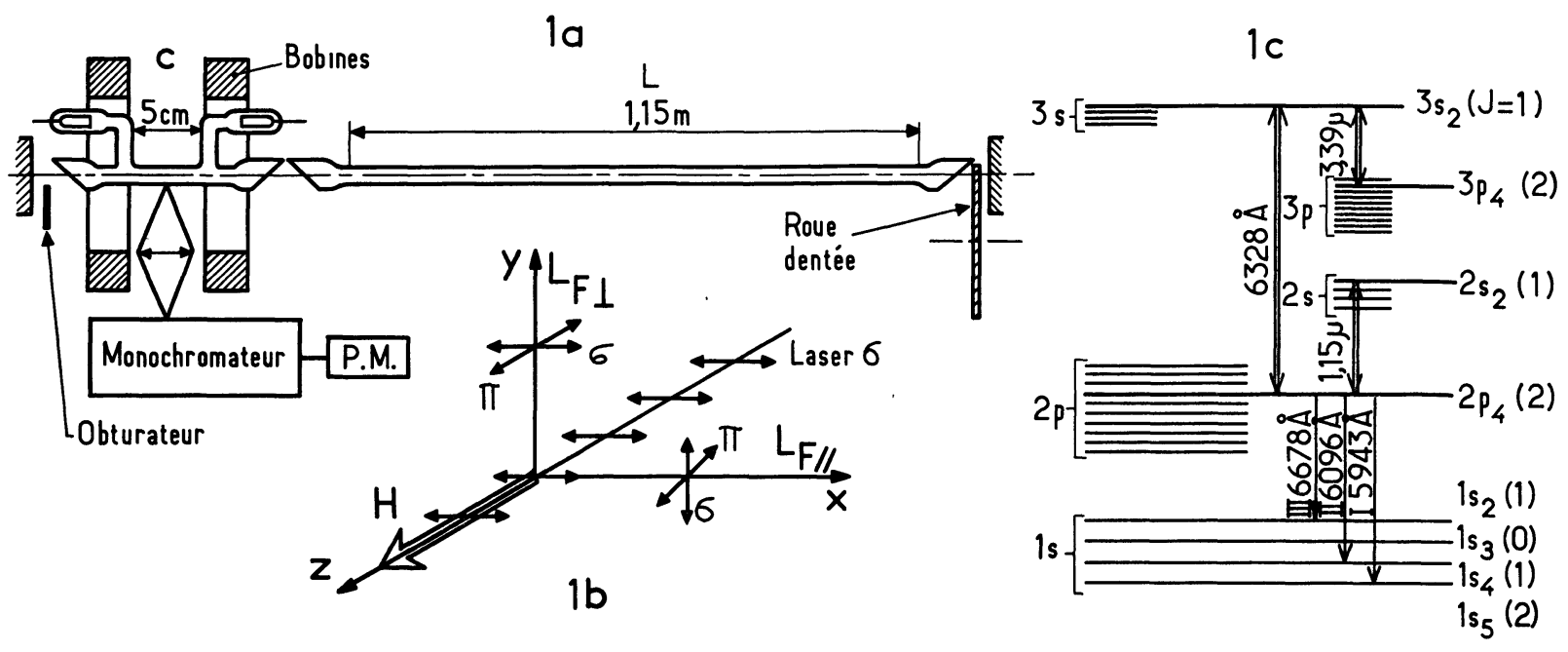

FIG. 1.

1 a) Montage expérimental; 1 b) Directions d'observation; 1 c) Niveaux d'énergie du néon.

Dans chaque direction d'observation, le faisceau lumineux traverse un polariseur $(\pi$ ou $\sigma)$ et les différentes raies sont isolées par un monochromateur suivi d'un photomultiplicateur (fig. $1 \mathrm{~b}$ ).

L'adjonction à ce montage d'une lame semi-transparente et d'un deuxième monochromateur dans chaque direction d'observation permet l'étude simultanée de deux raies de fluorescence.

Un obturateur placé à l'intérieur de la cavité permet de couper l'irradiation laser. On peut ainsi mesurer successivement le courant photoélectrique ${ }^{(0)} I$ en l'absence d'irradiation laser et la modification $\Delta I$ de ce courant provoquée par le laser.

Pour certaines mesures $(\Delta H$ et $R)$, seule la modification $\Delta I$ a de l'importance : il est donc avantageux d'atteindre directement cette modification par une modulation du faisceau laser. Une roue dentée entraînée par un moteur synchrone permet d'obturer périodiquement la cavité à une fréquence $\nu=130 \mathrm{c} / \mathrm{s}$. L'intensité du laser est alors modulée en créneaux de durée $\tau \simeq 4 \mathrm{~ms}$; les temps de descente et de montée des créneaux obtenus sont inférieurs à $\tau / 20$. La durée des créneaux est très supérieure à toutes les constantes de temps du système $\left(\sim 10^{-8} \mathrm{~s}\right.$ pour les atomes et $\sim 10^{-6} \mathrm{~s}$ pour la construction de l'onde laser), par suite la théorie stationnaire reste valable. Dans ces conditions, les raies de fluorescence issues de $a$ et $b$ sont également modulées en créneaux. A l'aide de détections synchrones, on mesure dans chaque direction d'observation l'amplitude $\Delta I$ de la modulation correspondante du courant photoélectrique.

L'introduction de la cellule $\mathrm{G}$ à l'intérieur de la cavité est intéressante à plusieurs titres :

1. G est soumise à la grande densité d'énergie lumineuse produite à l'intérieur de la cavité laser.
Les effets de l'irradiation sont, par suite, bien plus importants que si $\mathrm{C}$ se trouvait à l'extérieur du laser.

2. Cependant, les caractéristiques du milieu atomique de $\mathrm{C}$ et celles du rayonnement laser émis par $\mathrm{L}$ sont indépendantes, ce qui est avantageux à plusieurs points de vue :

a) Gette méthode nécessite seulement l'existence, dans C, d'une différence de population entre le niveau supérieur et le niveau inférieur de la transition laser. $\mathrm{C}$ n'est donc pas nécessairement amplificateur puisque le tube $\mathrm{L}$, lui, assure l'oscillation laser. La gamme de pressions et de mélanges que l'on peut étudier par cette méthode n'est donc limitée que par la possibilité d'obtenir une décharge.

b) Le champ magnétique appliqué sur C n'a aucune influence sur le fonctionnement du laser $\mathrm{L}$. L'expérience montre en effet que $\mathrm{C}$ perturbe très peu les caractéristiques de L. L'ensemble du tube amplificateur $L$ reste dans un champ très faible, même quand $\mathrm{C}$ est soumis à un champ de plusieurs dizaines de gauss.

On peut donc considérer comme fixes les caractéristiques du laser dans toutes les expériences où l'on fait varier le champ magnétique sur $\mathrm{C}$, alors que, dans des expériences comparables effectuées avec un seul tube laser soumis à un champ magnétique sur toute sa longueur, il est nécessaire de tenir compte, dans l'interprétation des résultats, de la réaction de $H$ sur l'oscillation laser.

B. Technique de mesure de $\Delta H^{(1)}, R^{(1)}$ et $F^{(1)}$. Influence de l'intensité du laser. - Dans $(I, E)$ nous avons caractérisé les modifications de la lumière de fluorescence sous l'effet de l'irradiation laser par les grandeurs $\Delta H, R, R^{\prime}$ et $F$ définies respectivement par 
les formules (I.62), (I.63), (I.68), (I.55). L'interprétation physique de ces grandeurs suppose que l'intensité de l'irradiation laser est uniforme dans toute la région de $\mathrm{C}$ dont on observe les raies de fluorescence. Or, cette hypothèse n'est pas vérifiée dans la pratique. De plus, certaines mesures sont basées sur la comparaison de l'intensité lumineuse émise dans les deux directions $\varphi=0$ et $\varphi=\pi / 2$. Il se pose donc un problème d'équilibrage entre les deux bancs de mesures.

Nous allons exposer dans ce paragraphe la méthode qui nous permet de donner une signification aux mesures.

Le courant photoélectrique $I$ mesuré dans chacune des directions est proportionnel à la somme des

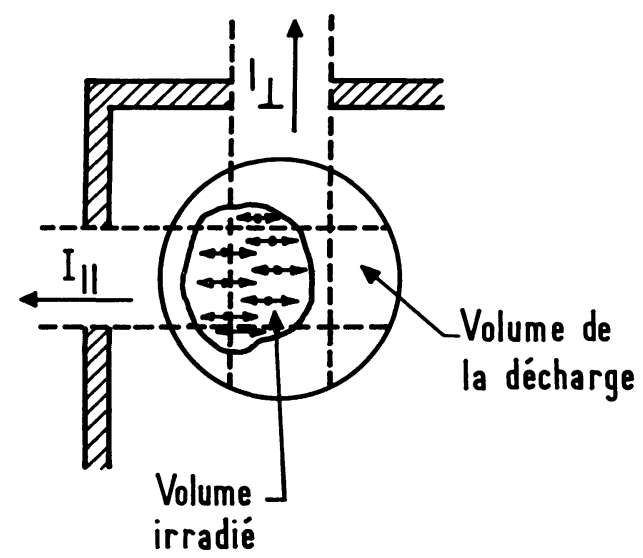

FIG. 2. - Les volumes observés dans la cellule $\mathrm{C}$.

contributions des volumes élémentaires vues par le système de détection ( fig. 2) :

$$
I=s \int_{V_{\text {obs. }}} L_{\mathrm{F}} \mathrm{d}^{3} r
$$

$s$ caractérise la sensibilité du système de mesure.

En particulier, on observe la lumière émise par certains atomes qui n'interagissent pas avec le faisceau laser. Par suite, la modification relative macroscopique de l'intensité de fluorescence, définie par :

$$
M=\frac{\Delta I}{(0) I}=\frac{\int_{V} \Delta L_{\mathrm{F}} \mathrm{d}^{3} r}{\int_{V}{ }^{(0)} L_{\mathrm{F}} \mathrm{d}^{3} r}
$$

n'a pas de signification physique précise : son ordre de grandeur constitue une borne inférieure de la modification microscopique $\Delta L_{\mathrm{F}} /{ }^{(0)} L_{\mathrm{F}}$ dans la région irradiée.

En champ fort, nous savons que le rayonnement émis est isotrope; cependant, les valeurs mesurées $M_{l l}$ et $M_{\perp}$ (dans les directions $\varphi=0$ et $\varphi=\pi / 2$ ) sont sensiblement différentes car les volumes observés $V_{\| \prime}$ et $V_{\perp}$ sont différents.
1. Mesure de $F^{(1)}$. - $F^{(\mathbf{1})}$ est défini par (I.56) comme la limite pour les faibles intensités du laser de la quantité :

$$
F=\frac{\Delta L_{\mathrm{F}}^{\pi}(1)}{\Delta L_{\mathrm{F}}^{\pi}(2)} \cdot \frac{{ }^{(0)} L_{\mathrm{F}}^{\pi}(2)}{\left({ }^{0}\right) L_{\mathrm{F}}^{\pi}(1)} \quad \text { (formule I.55) }
$$

(1) et (2) étant deux raies de fluorescence issues du même niveau ( $a$ ou $b$ ).

Pour la détermination de $F$, il est essentiel de comparer les raies de fluorescence émises par la même région de $\mathrm{C}$ et par suite observées dans la même direction, avec la même géométrie. Pour cela, on opère de la manière suivante : après la traversée d'un polariseur qui isole la composante $\pi$, la lumière est séparée en deux faisceaux par une lame semi-réfléchissante. Sur chacun des faisceaux, un monochromateur permet d'isoler l'une des raies. Grâce à l'interrupteur placé dans la cavité laser, on mesure les quantités ${ }^{(0)} I_{\pi}$ et $\Delta I_{\pi}$ simultanément sur les raies (1) et (2). On forme la quantité :

$$
F^{\prime}=\frac{\Delta I^{\pi}(1)}{\Delta I^{\pi}(2)} \frac{(0) I^{\pi}(2)}{(0) I^{\pi}(1)}=\frac{M^{\pi}(1)}{M^{\pi}(2)} .
$$

En réglant les deux monochromateurs sur la même raie, on vérifie que ce rapport est très peu sensible aux réglages optiques et reste égal à 1 à mieux de $1 \%$. Ceci montre que, dans ces conditions, les régions de $\mathrm{C}$ observées par les deux photomultiplicateurs sont bien identiques.

$F^{\prime}$ n'est pas en général égal à $F$ : pour qu'ils soient égaux, il faudrait que l'intensité du laser soit uniforme sur tout le volume irradié (alors $I=s L_{\mathrm{F}} V_{\text {irradie }}$ ). Cependant, nous allons montrer que $F^{\prime}$, comme $F$, tend vers $F^{(\mathbf{1})}$ quand l'intensité du laser tend vers zéro.

Supposons pour simplifier que l'on peut écrire, par unité de volume, ${ }^{(2)} L_{\mathrm{F}}={ }^{(2)} l_{\mathrm{F}} i_{\lambda}$ (approximation des très grandes largeurs Doppler). On a alors :

$$
\begin{gathered}
{ }^{(0)} I=s \int_{V}{ }^{(0)} L_{\mathrm{F}} \mathrm{d}^{3} r=s V^{(0)} L_{\mathrm{F}} \\
\left.\Delta I=s \int_{V}{ }^{(2)} L_{\mathrm{F}}+{ }^{(4)} L_{\mathrm{F}}+\ldots\right) \mathrm{d}^{3} r \\
\left.=s^{(2)} l_{\mathrm{F}} \int_{V} i_{\lambda}(\mathbf{r}) \mathrm{d}^{3} r+s \int_{V}{ }^{(4)} L_{\mathrm{F}}+\ldots\right) \mathrm{d}^{3} r .
\end{gathered}
$$

Dans ces conditions :

$$
\underset{i \lambda \rightarrow 0}{\operatorname{Limite}} F^{\prime}=\frac{(2) l_{\mathrm{F}}^{\pi}(1)}{{ }^{(2)} l_{\mathrm{F}}^{\pi}(2)} \frac{(0) L_{\mathrm{F}}^{\pi}(2)}{\left({ }^{(0)} L_{\mathrm{F}}(1)\right.} \equiv F^{(1)} .
$$

Nous avons vérifié expérimentalement que cette détermination de $F^{(\mathbf{1})}$ est alors bien indépendante de la direction d'observation en effectuant simultanément ces mesures dans les directions $\varphi=0$ et $\varphi=\pi / 2$. Les résultats obtenus sont identiques à mieux que $1 \%$. 


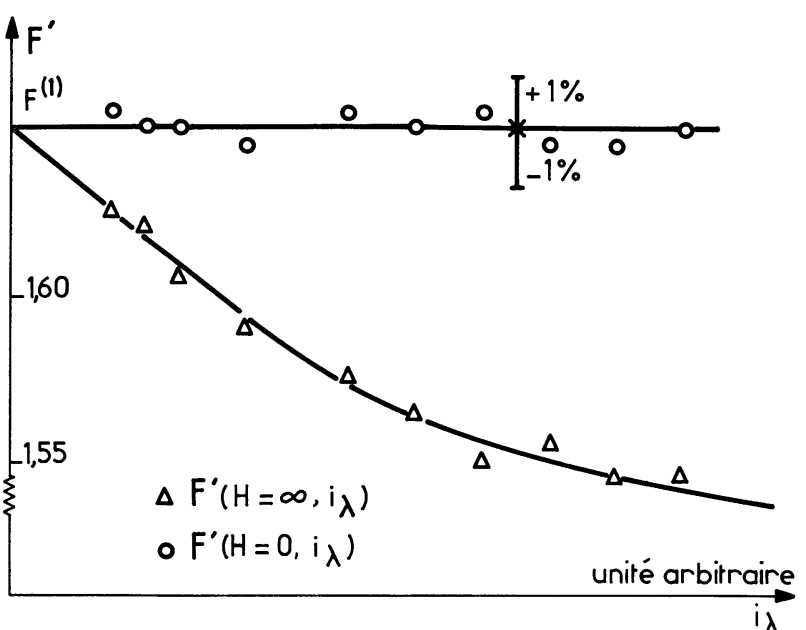

FIG. 3. - Déterminations de $F^{\prime}(\mathrm{I}$, II) relatives au niveau $2 p_{4}$ en fonction de l'intensité du laser $(80 \% \mathrm{He}$, $20 \% \mathrm{Ne}, p=1,55$ torr).

La figure 3 montre un exemple de variation de $F$ en fonction de $i_{\lambda}$ pour le niveau $2 p_{4}$ du néon. Sur le même graphique, nous avons porté $F^{\prime}(H=0)$ et $F^{\prime}(H=\infty)$. Il apparaît que $F^{\prime}(H=0)$ est indépendant de l'intensité du laser. Ceci est général à mieux que $1 \%$ près pour toutes les mesures faites avec le niveau $2 p_{4}$. Cette remarque nous a permis de simplifier la méthode de mesure pour ce niveau puisque, à la précision de nos mesures, $F^{\prime}(H=0)=F^{(\mathbf{1})}$.

2. Mesure De $R^{(1)}$ ET DE $\Delta H^{(1)}$. - La largeur $\Delta H$ (I.62) et la hauteur relative $R$ (I.63) de l'effet Hanle ne font intervenir que les changements d'intensité de fluorescence $\sigma$ dus à l'irradiation laser, il ne se pose donc qu'un problème d'équilibrage entre les deux voies.

En effet, en utilisant (II.5), on voit aisément que

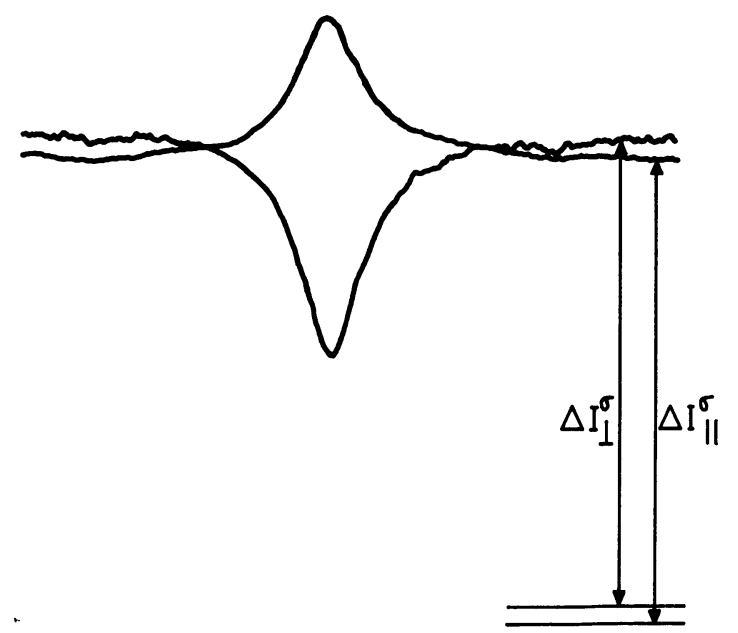

FIG. 4. - Enregistrement typique sur la raie $\lambda=5943 \AA\left(2 p_{4}-1 s_{5}\right)$ en fonction du champ $H$. les grandeurs macroscopiques $R_{/ /}$et $R_{\perp}$ formées à partir de $\Delta I^{\sigma}$ et les grandeurs microscopiques correspondantes formées à partir de $\Delta L^{\sigma}$ ne diffèrent que par des termes du $2^{\mathrm{e}}$ ordre en $i_{\lambda}$. Leur limite $R^{(\mathbf{1})}$ est donc la même.

De plus, il est possible de régler les sensibilités $s_{/ /}$et $s_{\perp}$ des détecteurs de façon à égaliser les valeurs de $\Delta I_{I I}^{\sigma}$ et $\Delta I_{\perp}^{\sigma}$ en champ fort (lorsque $\Delta L^{\sigma}$ est isotrope). Dans ces conditions, la comparaison entre $\Delta I_{\| /}^{\sigma}$ et $\Delta I_{\perp}^{\sigma}$ a une signification (au moins au second ordre) (fig. 4).

Avec cette normalisation, la mesure de $\Delta I_{I I}-\Delta I_{\perp}$ élimine toute variation isotrope et augmente sensiblement le rapport signal sur bruit de la variation anisotrope en compensant les fluctuations identiques dans les deux directions (fluctuations identiques de la décharge de $\mathrm{C}$, fluctuations de tension de l'alimentation commune aux deux photomultiplicateurs). Cette méthode est la plus précise pour vérifier la forme de raie lorentzienne des courbes d'effet Hanle et pour mesurer la largeur à mi-hauteur $\Delta H$. Elle a été utilisée de manière systématique dans ce but. La différence $\Delta I_{I /}^{\sigma}-\Delta I_{\perp}^{\sigma}$ est construite électroniquement, soit directement à partir du courant fourni par les photomultiplicateurs (méthode continue), soit à partir du signal donné par les détections synchrones (méthode par modulation du laser). Les deux méthodes sont sensiblement équivalentes pour la mesure de $\Delta H$.

Une étude systématique de l'effet Hanle du niveau $3 s_{2}$ irradié alternativement par le laser $\lambda=6328 \AA$ et le laser $\lambda=3,39 \mu$ a montré tout d'abord que $\Delta H$ varie linéairement avec la puissance du laser tant que cette dernière est relativement faible ( fig. 5). D'autre part, la valeur extrapolée $\Delta H^{(\mathbf{1})}$ pour les intensités de

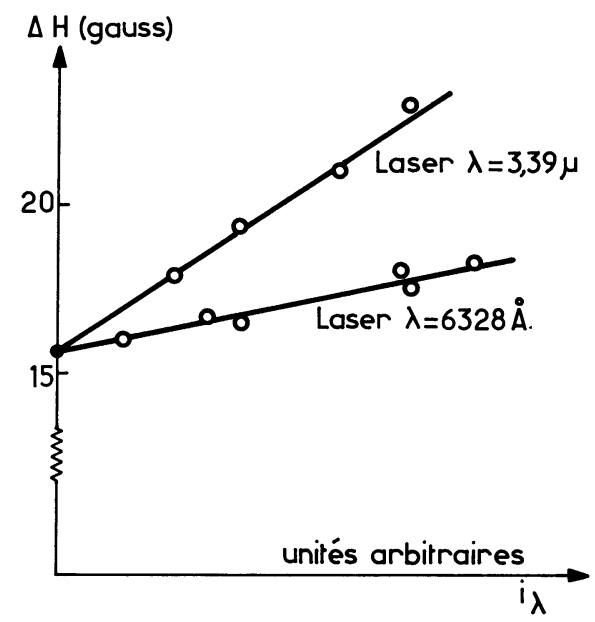

FIG. 5. - Effet de l'intensité d'irradiation sur la largeur $\Delta H$ de l'effet Hanle du niveau $3 s_{2}(10 \% \mathrm{Ne}$, $90 \% \mathrm{He}, p=1,2$ torr).

laser évanescentes ne dépend pas de la raie laser utilisée : conformément à la théorie, $\Delta H^{(1)}$ est caractéristique du temps de relaxation de l'alignement 
transversal sous la seule influence des paramètres définissant le milieu atomique de $\mathrm{C}$. Cet effet d'élargissement peut atteindre plusieurs gauss $(\sim 20 \%$ de la largeur) avec la raie $\lambda=6328 \AA$. Cette extrapolation est par suite absolument nécessaire pour l'obtention d'une mesure précise des relaxations. Elle a été faite de manière systématique dans toutes les mesures dont nous parlerons par la suite.

Pour la mesure de $R_{/ /}$et $R_{\perp}$, la méthode de modulation $\mathrm{du}$ laser donne une bien meilleure précision que la méthode continue. Pour cela, on enregistre simultanément le signal $\Delta I_{\mid /}$et $\Delta I_{\perp}$ fourni par les détections synchrones et on utilise la définition (I.63) pour former $R_{/ /}$et $R_{\perp}$. Il n'est pas nécessaire pour cette mesure d'équilibrer parfaitement les deux voies.

A partir de $R_{/ /}$et $R_{\perp}$, on forme $R$ et $R^{\prime}$ (formules (I.67) et (I.68)). On pourrait mesurer directement le taux d'anisotropie en champ nul $R^{\prime}$, mais ceci nécessite un excellent équilibrage des deux voies. La construction de $R^{\prime}$ à partir de $R_{\mid /}$et $R_{\perp}$ est plus rapide et plus précise. Les valeurs de $R_{/ /}$et $R_{\perp}$ pour les différentes raies de fluorescence issues du niveau $3 s_{2}$ sont trop faibles pour qu'une étude précise ait pu être envisagée sur ce niveau. Pour le niveau $2 p_{4}$, par contre, il est possible de vérifier que $R_{/ /}$et $R_{\perp}$ dépendent effectivement de l'intensité d'irradiation $i_{\lambda}$.

La figure 6 montre la variation des valeurs absolues de $R_{\| l}, R_{\perp}, R$ et $R^{\prime}$ en fonction de l'intensité du

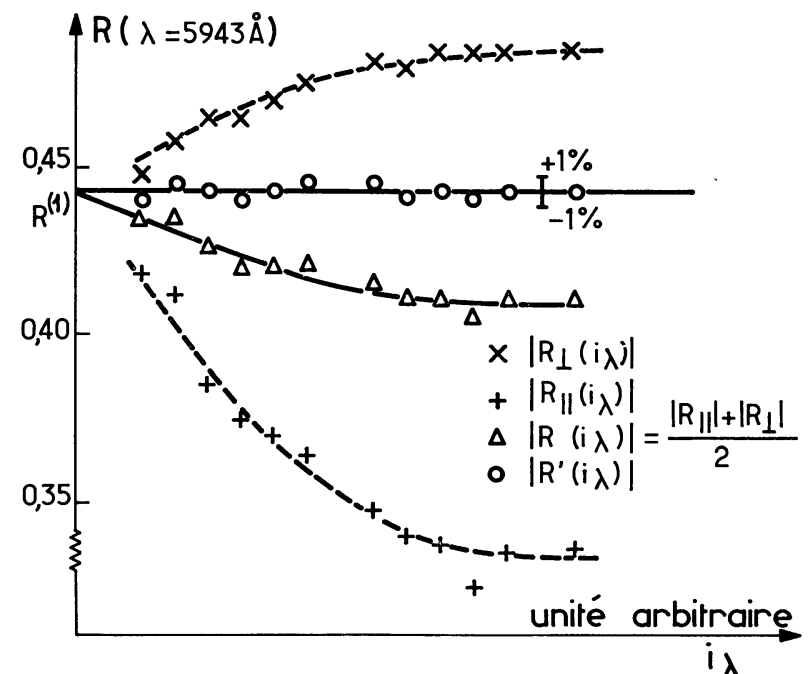

FIG. 6. - Déterminations de $R \|, R_{\perp}, R$ et $R^{\prime}$ en fonction de l'intensité du laser $(80 \% \mathrm{He}, 20 \% \mathrm{Ne} ; p=1,30$ torr; $i_{\mathrm{c}}=7 \mathrm{~mA}$; niveau $2 p_{4}$; raie $\lambda=5943 \AA$ ).

laser $i_{\lambda}$. On voit sur cette figure l'utilité de construire $R$ et $R^{\prime}$ : alors que $R_{/ /}$et $R_{\perp}$ varient beaucoup avec $i_{\lambda}$ et ne permettent pas une extrapolation précise, $R$ et $R^{\prime}$ ont une variation faible et donnent $R^{(\mathbf{1})}$ avec une bonne précision.

De plus, $R^{\prime}$ peut être considéré comme indépendant de $i_{\lambda}$ à la précision de nos mesures, c'est-à-dire à mieux que $1 \%$ près. Ce fait a été vérifié dans de nombreux cas et il nous a permis de simplifier appréciablement la méthode de mesure de $R^{(1)}$ (pour le niveau $\left.2 p_{4}\right)$.

Dans toute la suite, nous partirons des valeurs $\Delta H^{(\mathbf{1})}$, $R^{(\mathbf{1})}$ et $F^{(\mathbf{1})}$ obtenues de cette manière. Cette extrapolation de $\Delta H, R$ et $F$, qui permet de s'affranchir des effets radiatifs d'élargissement et de dépolarisation par l'onde laser, élimine par ce fait même les couplages provoqués par l'émission induite entre le niveau supérieur et le niveau inférieur.

Les caractéristiques $\Delta H^{(\mathbf{1})}, R^{(\mathbf{1})}$ et $F^{(\mathbf{1})}$ d'un niveau supérieur d'une transition laser doivent donc être attribuées à ce seul niveau. Nous savons (I, E) qu'il n'en est pas forcément de même pour le niveau inférieur en raison des transferts provenant du niveau supérieur par émission spontanée.

L'étude détaillée du niveau supérieur $3 s_{2}$ de la transition laser $\lambda=6328 \AA$ fera l'objet d'une publication ultérieure [9]. Nous nous contenterons de présenter les résultats de cette étude indispensables à l'interprétation des mesures effectuées sur le niveau inférieur $2 p_{4}$.

C. Le niveau $2 p_{4}$ du néon. - Le niveau $2 p_{4}$ de moment cinétique $J_{\mathrm{a}}=2$ est le niveau inférieur $a$ de la transition $\lambda=6328 \AA\left(3 s_{2}-2 p_{4}\right)$. Le moment cinétique $J_{\mathrm{b}}$ du niveau supérieur $3 s_{2}$ est égal à 1 .

Le niveau $2 p_{4}$ possède trois raies de fluorescence, repérées dans toute la suite par les indices I, II et III de longueurs d'onde suivantes :

$$
\begin{gathered}
\lambda_{\mathrm{I}}=5943 \AA\left(2 p_{4}-1 s_{5}\right) \\
\lambda_{\text {II }}=6096 \AA\left(2 p_{4}-1 s_{4}\right) \text { et } \lambda_{\text {III }}=6678 \AA\left(2 p_{4}-1 s_{2}\right) .
\end{gathered}
$$

Il est intéressant de comparer les résultats des mesures effectuées sur la raie I (qui aboutit sur un niveau $J_{\mathrm{f}}=2$ ) à ceux obtenus sur les raies II ou III $\left(J_{f^{\prime}}=1\right)$. La raie III, inséparable d'une raie intense de l'hélium, présente dans la plupart de nos expériences, n'a pas été étudiée de façon systématique.

1. L'EFFET DU TRANSFERT PAR ÉMISSION SPONTANÉE. - En raison du couplage avec le niveau $3 s_{2}$ dû à l'émission spontanée, l'interprétation des résultats de $R^{(1)}, F^{(\mathbf{1})}$ et $\Delta H^{(1)}$ relatifs au niveau $2 p_{4}$ suppose la connaissance préalable des caractéristiques du niveau $3 s_{2}$.

En reportant dans les formules (I.70) les valeurs numériques $\left(a \equiv 2 p_{4}, b \equiv 3 s_{2}\right)$, on voit que la mesure de $F^{(\mathbf{1})}$ et de $R^{(\mathbf{1})}$ permet d'atteindre les quantités :

$$
\begin{gathered}
\Gamma_{a}(0)\left[1-\frac{\gamma_{b a}}{\Gamma_{b}(0)}\right]^{-1} \\
\Gamma_{a}(2)\left[1-\frac{\gamma_{b a}}{\Gamma_{b}(0)} \cdot \frac{\Gamma_{b}(0)}{10 \Gamma_{b}(2)}\right]^{-1} .
\end{gathered}
$$


De même, la correction à apporter à la forme de Lorentz de l'effet Hanle est en valeur relative de l'ordre de $\frac{\gamma_{b a}}{\Gamma_{b}(0)} \cdot \frac{\Gamma_{b}(0)}{10 \Gamma_{b}(2)}$.

Or, l'étude précise du niveau $3 s_{2}$ à partir de l'irradiation par la raie $\lambda=6328 \AA\left(3 s_{2}-2 p_{4}\right)$ est rendue difficile par la faible valeur des alignements créés. Elle a néanmoins permis d'atteindre un ordre de grandeur des paramètres du niveau $3 s_{2}$.

a) Transfert d'alignement. - En tenant compte de la diffusion multiple cohérente, on trouve que le rapport $\Gamma_{b}(0) / \Gamma_{b}(2)$ est au plus de l'ordre de $1 / 2$ dès que la pression partielle de néon est suffisante pour que la raie de résonance $3 s_{2}-1 p_{0}$ soit totalement réabsorbée. Ceci se produit sûrement dès que $P_{\mathrm{Ne}}>200 \mu$. Dans ce cas, l'effet du transfert par émission spontanée est au moins vingt fois plus faible pour l'alignement que pour la composante scalaire. Nous verrons plus loin que ce transfert entraîne une correction relativement faible sur la population du niveau $2 p_{4}$. Il est par suite légitime de négliger complètement son effet sur les composantes de l'alignement.

Ceci implique en particulier que l'effet Hanle observé sur I, II et III est uniquement dû à l'interaction directe du rayonnement laser avec le niveau $2 p_{4}$, le transfert d'effet Hanle à partir du niveau $3 s_{2}$ apportant une correction négligeable. Ce fait a pu être vérifié expérimentalement de la manière suivante :

L'irradiation par un laser à la longueur d'onde $\lambda=3,39 \mu\left(3 s_{2}-3 p_{4}\right)$ d'intensité convenable entraîne sur le niveau $3 s_{2}$ le même changement de population et la même proportion d'alignement que l'irradiation à la longueur d'onde $\lambda=6328 \AA\left(3 s_{2}-2 p_{4}\right)$. L'irradiation à la longueur d'onde $\lambda=3,39 \mu$ est par contre sans effet direct sur le niveau $2 p_{4}$ et les modifications observées alors sur I, II ou III sont uniquement dues aux transferts par émission spontanée. Dans ces conditions, aucun effet Hanle n'est détectable sur le niveau $2 p_{4}$. Il est donc certain que la largeur $\Delta H^{(1)}$ de l'effet Hanle observée sur I, II ou III en présence de l'irradiation à la longueur d'onde $\lambda=6328 \AA$ est reliée à la seule largeur $\Gamma_{\mathrm{a}}(2)$.

Une preuve supplémentaire de ce fait est donnée par la comparaison des largeurs $\Delta H^{(\mathbf{1})}$ obtenues dans les mêmes conditions de pression et de décharge lors de l'irradiation successive par les deux raies laser : $\lambda=6328 \AA\left(3 s_{2}-2 p_{4}\right)$ et $\lambda=1,15 \mu\left(2 s_{2}-2 p_{4}\right)$ (pour laquelle $\left.J_{\mathrm{a}}=2, J_{\mathrm{b}}=1\right)$. Le transfert par émission spontanée est sensiblement plus important à partir du niveau $2 s_{2}$ qu'à partir du niveau $3 s_{2}$. Néanmoins, comme le montre la figure 7 , les largeurs $\Delta H^{(1)}$ déduites des deux expériences sont identiques. Cette expérience qui constitue pour le niveau $2 p_{4}$ le pendant de celle décrite en (II.B.2) pour le niveau $3 s_{2}$ est faite avec une précision de l'ordre de $1 \%$. Elle montre que l'on peut négliger les transferts d'alignement dans ce cas. Il résulte de tout ceci que le terme de transfert peut être négligé pour les alignements ${ }_{a} \rho_{0}^{2}$ et ${ }_{a} \rho_{2}^{2}$ dans

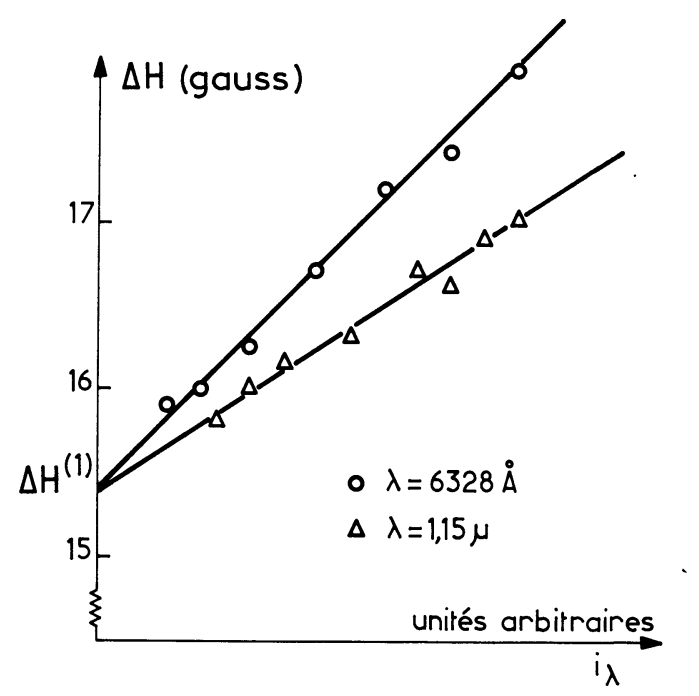

FIG. 7. - Effet de l'intensité d'irradiation sur la largeur $\Delta H$ de l'effet Hanle du niveau $2 p_{4}(80 \% \mathrm{He}$, $20 \% \mathrm{Ne}, p=1,20$ torr, $i_{\mathrm{c}}=15 \mathrm{~mA}$ ).

les formules (I.47.a). Il n'est donc pas nécessaire de faire la correction (II.8) sur la mesure de $\Gamma_{a}(2)$. Cette simplification est due aux valeurs particulières de $J_{\mathrm{a}}$ et $J_{\mathrm{b}}$ pour lesquelles (II .8) contient un coefficient $1 / 10$. Elle ne saurait être généralisée dans le cas d'une raie laser de $J_{\mathrm{a}}$ et $J_{\mathrm{b}}$ quelconques.

b) Transfert de population. - L'irradiation avec le laser $\lambda=3,39 \mu$ ( $\{$ G.1.a) prouve que le transfert de population $\left(3 s_{2} \rightarrow 2 p_{4}\right)$ n'est pas négligeable. De plus, les irradiations laser $\lambda=1,15 \mu$ et $\lambda=6328 \AA$ conduisent à des valeurs très différentes de $R^{(\mathbf{1})}$ et $F^{(\mathbf{1})}$ : comme ces mesures ont été faites dans les mêmes conditions (pression, décharge, mélange), il faut en conclure que la différence vient du transfert de population.

Il est donc nécessaire de connaître le rapport $\gamma_{\mathrm{ba}} / \Gamma_{\mathrm{b}}(0)$. La probabilité de transition $\gamma_{\mathrm{ba}}$ est constante, tandis que la relaxation $\Gamma_{b}(0)$ dépend des conditions expérimentales, en particulier des collisions et de la diffusion multiple sur la raie de résonance $3 s_{2}-1 p_{0}$. Si $\lambda$ est le rapport de branchement de cette raie $\left(\lambda=\gamma_{3 s_{2} \rightarrow 1 p_{0}} / \gamma_{\mathrm{b}}\right)$ et $x$ son taux de réabsorption, la formule (I.12) donne :

$$
\begin{aligned}
& \Gamma_{\mathrm{b}}(0)=\left(\Gamma_{\mathrm{b}}(0)\right)_{\mathrm{rad} .}+\left(\Gamma_{\mathrm{b}}(0)\right)_{\text {coll. }} \\
& =\gamma_{\mathrm{b}}(1-\lambda x)+\alpha_{\mathrm{b}} p_{\mathrm{Ne}}+\beta_{\mathrm{b}} p_{\mathrm{He}} .
\end{aligned}
$$

L'étude directe du niveau $3 s_{2}$ [9] permet de donner l'ordre de grandeur de $\alpha_{b}$ et $\beta_{b}$. D'autre part, dès que la pression partielle de néon est supérieure à $200 \mu$, la réabsorption de la raie de résonance est totale $(x=1)$. A l'exception du cas du mélange à $1 \%$ de néon (cf. $\S$ G.3.c), cette condition est pratiquement toujours réalisée. On peut donc écrire :

$$
\left(\Gamma_{\mathrm{b}}(0)\right)_{\mathrm{rad} .} \simeq \gamma_{\mathrm{b}}(1-\lambda)
$$


$\left(\Gamma_{\mathbf{b}}(0)\right)_{\text {rad. }}$ peut alors être considéré comme constant. Il représente la probabilité d'émission spontanée sur toutes les raies de fluorescence autres que la raie de résonance. G'est d'ailleurs l'existence même de ces processus d'émission spontanée à côté de l'émission sur la raie de résonance qui permet d'obtenir un alignement du niveau $3 s_{2}$, bien que la diffusion multiple cohérente de la raie de résonance soit complètement saturée. Ce cas est tout à fait comparable à celui du niveau $3 P_{1}^{(0)}$ du plomb étudié par E. B. Saloman et E. W. Happer [5].

On peut alors définir le facteur de transfert :

$$
\begin{array}{r}
\varepsilon=\frac{\gamma_{\mathrm{ba}}}{\Gamma_{\mathrm{b}}(0)}=\frac{\gamma_{\mathrm{ba}}}{\left(\Gamma_{\mathrm{b}}(0)\right)_{\mathrm{rad} .}} \times \frac{\left(\Gamma_{\mathrm{b}}(0)\right)_{\mathrm{rad} .}}{\left(\Gamma_{\mathrm{b}}(0)\right)_{\mathrm{rad} .}+\left(\Gamma_{\mathrm{b}}(0)\right)_{\mathrm{coll}} .} \\
=\frac{\varepsilon_{0}}{1+0,35 p_{\mathrm{Ne}}+0,08 p_{\mathrm{He}}}
\end{array}
$$

les pressions partielles étant exprimées en torrs.

Dans ces conditions, pour les raies I et II, $R^{(1)}$ et $F^{(1)}$ deviennent (en utilisant (I.66), (I.58), (II.7) et (II.11)) :

$$
\begin{aligned}
R_{(\text {I ou II })}^{(1)} & =\frac{21 \Gamma_{\mathrm{a}}(0)}{40 \Gamma_{\mathrm{a}}(2)(1-\varepsilon) \mp 7 \Gamma_{\mathrm{a}}(0)} \\
F^{(1)}(\mathrm{I}, \mathrm{II}) & =\frac{20 \Gamma_{\mathrm{a}}(2)(1-\varepsilon)+7 \Gamma_{\mathrm{a}}(0)}{20 \Gamma_{\mathrm{a}}(2)(1-\varepsilon)-7 \Gamma_{\mathrm{a}}(0)} .
\end{aligned}
$$

On peut prévoir que l'existence du transfert augmente les valeurs de $R^{(1)}$ et de $F^{(1)}$. Il est intéressant de poser :

$$
\begin{gathered}
A_{(\mathrm{I} \mathrm{ou} \mathrm{II})}=\frac{1}{R_{(\mathrm{I} \mathrm{ou} \mathrm{II})}}-\frac{40 \mp 7}{21} \\
A_{(\mathrm{I} \text { ou II })}^{(1)}=\operatorname{limite}_{i \lambda \rightarrow 0} A_{(\mathrm{I} \text { ou II })}=\frac{1}{R_{(\mathrm{I} \text { ou II })}^{(1)}}-\frac{40 \mp 7}{21} \\
=\frac{40}{21}\left[(1-\varepsilon) \frac{\Gamma_{\mathrm{a}}(2)-\Gamma_{\mathrm{a}}(0)}{\Gamma_{\mathrm{a}}(0)}-\varepsilon\right] .
\end{gathered}
$$

En effet, $A^{(1)}$ est en théorie indépendant de la raie de fluorescence. D'autre part, à faible pression, $\Gamma_{\mathrm{a}}(2)$ et $\Gamma_{\mathrm{a}}(0)$ tendent tous deux vers $\gamma_{\mathrm{a}}=1 / \tau_{\mathrm{a}}$ et la mesure de $A$ donne :

$$
\underset{p \rightarrow 0}{\operatorname{limite}} A=-\frac{40}{21} \varepsilon_{0} \text {. }
$$

Il est également possible de trouver $\varepsilon_{0}$ par une mesure totalement indépendante :

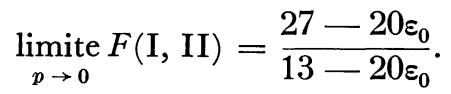

Remarque : Dans cette analyse, nous avons négligé tous les transferts d'excitation du niveau $3 s_{2}$ au niveau $2 p_{4}$ plus complexes que le processus direct sur la raie $6328 \AA$. Un transfert de population en trois étapes $\left(3 s_{2} \rightarrow 3 p_{\mathrm{n}} ; 3 p_{\mathrm{n}} \rightarrow 2 s_{\mathrm{m}} ; 2 s_{\mathrm{m}} \rightarrow 2 p_{4}\right)$ peut exister. Cet effet ne constitue qu'un terme correctif qui affecte seulement la détermination de $\gamma_{b a}$ que l'on peut déduire de la connaissance de $\varepsilon_{0}$. Une estimation expérimentale de la modification de population des niveaux $2 s$ provoquée par le laser 6328 conduit à une correction inférieure à $2 \%$ sur $\gamma_{\mathrm{ba}}$.

2. REGHERGHe DES PARAMÈTRES AGISSANT SUR $\Delta H^{(1)}$, $R^{(1)}$ ET $F^{(1)}$. LES EXTRAPOLATIONS SUCGESSIVES. a) L'analyse simultanée des raies I et II permet plusieurs confrontations :

1) On peut mesurer simultanément $\Delta H$ sur les deux raies. Ceci conduit au même résultat à mieux que $1 \%$ près. Il en est bien entendu de même pour $\Delta H^{(1)}$.

2) On peut mesurer $R_{\mathrm{I}}^{(1)}$ et $R_{\mathrm{II}}^{(1)}$ : on vérifie bien que la quantité $A^{(1)}$ formée à partir de ces grandeurs est indépendante de la raie de fluorescence (fig. 8). Geci constitue une vérification d'une hypothèse impor-

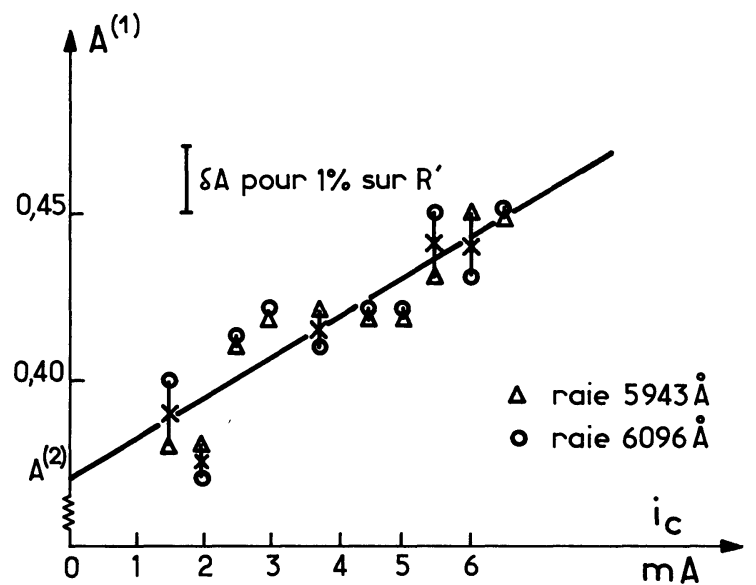

FIG. 8. - Comparaison des deux déterminations de $A^{(\mathbf{1})}$ sur les raies I et II ( $80 \% \mathrm{He}, 20 \% \mathrm{Ne}, p=0,95$ torr $)$.

tante de la théorie : si la disparition de l'effet Hanle en l'absence d'irradiation laser prouve bien que la décharge ne crée pas d'alignement transversal, l'identité des deux déterminations simultanées de $A$ constitue la vérification la plus sensible de l'hypothèse, suivant laquelle la décharge ne crée pas non plus d'alignement longitudinal $\left({ }^{3}\right)$.

b) On constate que les grandeurs $\Delta H^{(1)}, R_{\mathrm{I}}^{(1)}, R_{\mathrm{II}}^{(1)}$ (ou $A^{(1)}$ ) et $F^{(1)}(\mathrm{I}, \mathrm{II})$ dépendent sensiblement des paramètres suivants :

- la composition du mélange d'hélium et de néon contenu dans la cellule $\mathrm{C}$;

- la pression totale de ce mélange;

- l'intensité $i_{\mathrm{c}}$ de la décharge excitatrice.

(3) En toute rigueur, si l'alignement n'est pas nul, on démontre que l'égalité des deux mesures de $A$ ne peut être réalisée que si le rapport entre l'alignement créé par la décharge dans les niveaux $a$ et celui créé dans le niveau $b$ a une valeur déterminée. Il faudrait donc que ce rapport ait juste la bonne valeur quelles que soient les conditions de pression, de mélange et de décharge, ce qui est hautement improbable puisque les populations sont très sensibles à tous ces paramètres. 
Pour faire varier indépendamment ces trois paramètres, nous avons été amenés à opérer systématiquement de la manière suivante :

On remplit la cellule $\mathrm{G}$ à partir d'une bouteille contenant un mélange d'hélium et de néon en proportions fixes. En conservant la pression constante dans la cellule, on fait un ensemble de mesures pour différentes valeurs de l'intensité $i_{\mathrm{c}}$ de la décharge. On constate alors pour des intensités de décharge de quelques milliampères que $\Delta H^{(1)}, R_{\mathrm{I}}^{(1)}$ et $R_{\mathrm{II}}^{(1)}$ varient linéairement avec $i_{\mathrm{c}}$, alors que $F^{(1)}$ reste pratiquement invariant. Nous étudierons en (II.c.4) l'origine de ces effets du courant de la décharge. Les valeurs $\Delta H^{(2)}$, $R_{\mathrm{I}}^{(2)}, R_{\mathrm{II}}^{(2)}$ et $F^{(2)}$ extrapolées aux intensités de décharges évanescentes sont caractéristiques des relaxations sous l'effet de l'émission spontanée et des collisions avec les atomes dans l'état fondamental.

Il suffit de reprendre cette double extrapolation $\left(i_{\lambda}\right.$ et $\left.i_{\mathrm{c}}\right)$ à différentes valeurs de la pression pour déterminer l'effet des collisions dans le mélange. Pour obtenir indépendamment l'effet des collisions du néon et de l'hélium, il faut recommencer avec de nouveaux mélanges.

Nous avons fait des mesures systématiques sur 4 mélanges :

$1 \%$ de néon-99\% d'hélium

$10 \%$ de néon-90\% d'hélium

$20 \%$ de néon- $80 \%$ d'hélium

$100 \%$ de néon- $0 \%$ d'hélium

et pour une vingtaine de pressions dans chaque cas.

3. L'Étude des gollisions. Détermination Quantitative De L'effet de Gascade. - a) $\Delta H^{(2)}$. Mesure de $\Gamma_{\mathrm{b}}(2)$. - On constate expérimentalement que, pour chaque valeur de la composition du mélange d'hélium et de néon de la cellule $\mathrm{C}, \Delta H^{(2)}$ varie linéairement avec la pression (fig. 9). D'autre part, le point extrapolé $\Delta H^{(0)}$ aux pressions évanescentes est bien indépendant de la composition du mélange. Ce point commun correspond à la largeur naturelle du niveau $\gamma_{a}$ qui permet le calcul de la durée de vie [6] :

$\tau_{2 p_{4}}=\frac{1}{\gamma_{\mathrm{a}}}=\frac{1}{g \beta \Delta H^{(0)}}=(1,70 \pm 0,04) 10^{-8} \mathrm{~s}$.

La pente $\frac{1}{P} \frac{\Delta H^{(2)}-\Delta H^{(0)}}{\Delta H^{(0)}}$ où $P$ est la pression du mélange représente physiquement la variation relative du temps de relaxation de l'alignement due aux collisions. On constate que c'est une fonction linéaire de la proportion d'hélium et de néon de la cellule (fig. 10). $\Gamma_{\mathrm{a}}(2)$ s'exprime de la manière suivante :

$$
\begin{aligned}
\Gamma_{\mathrm{a}}(2)=\gamma_{\mathrm{a}}\left[1+(0,580 \pm 0,005] P_{\mathrm{He}}\right. & \\
& \left.+(0,360 \pm 0,005) P_{\mathrm{Ne}}\right]
\end{aligned}
$$

les pressions partielles $P_{\mathrm{He}}$ et $P_{\mathrm{Ne}}$ étant exprimées en torrs.

En admettant qu'il règne dans la cellule une température de $300^{\circ} \mathrm{K}$, ceci conduit à des sections efficaces

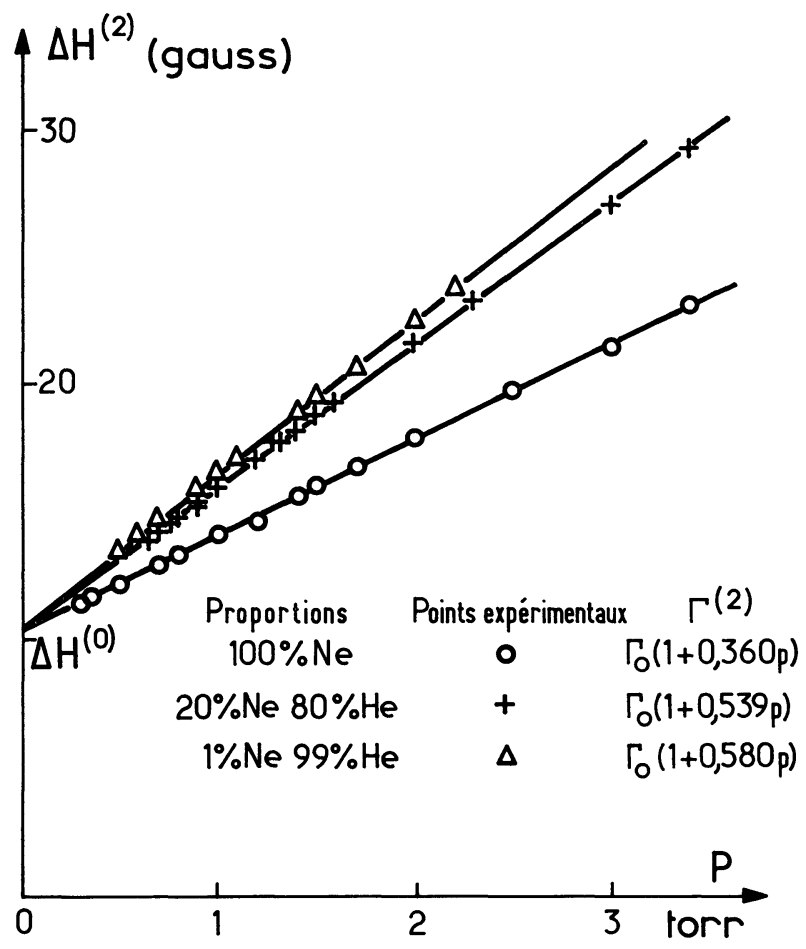

FIG. 9.

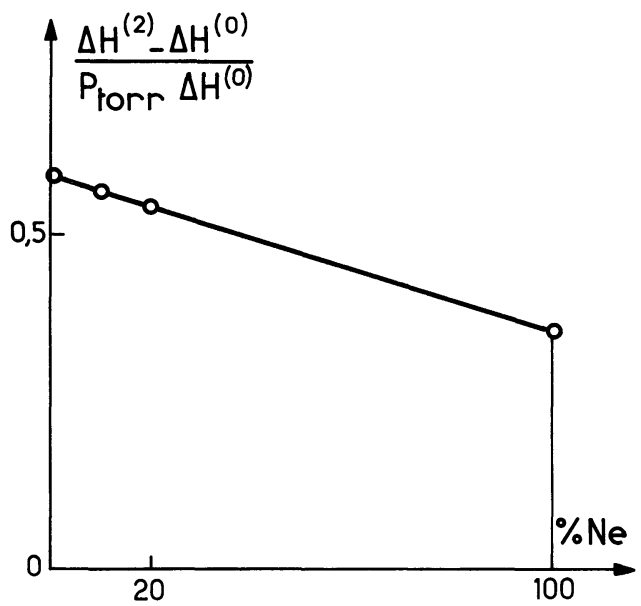

FIG. 10.

de collisions (avec la définition de Mitchell et Zemansky) [7] qui prennent les valeurs suivantes :

$$
\begin{aligned}
& \sum_{\mathrm{Ne}-\mathrm{Ne}^{*}}(2)=(0,74 \pm 0,02) 10^{-14} \mathrm{~cm}^{2} \\
& \sum_{\mathrm{He}-\mathrm{Ne}^{*}}(2)=(0,72 \pm 0,02) 10^{-14} \mathrm{~cm}^{2} .
\end{aligned}
$$

Ces valeurs sont très comparables. Rappelons que la transition $\mathrm{Ne}\left(2 p_{4}\right) \rightarrow \mathrm{Ne}$ (fondamental) est interdite. Dans des cas similaires, les sections efficaces d'hélium et de néon ont toujours été trouvées très voisines [8]. 
La précision donnée ici est assez illusoire car on ne connaît pas la température du mélange à l'intérieur de la cellule (après extrapolation à $i_{\mathrm{c}}$ nul).

b) $A^{(2)}$ et $F^{(2)}$. Mesure de $\Gamma_{\mathrm{a}}(0)$ et $\varepsilon$. — Dans l'exposé des résultats relatifs aux mesures de $A^{(2)}$ et $F^{(2)}(\mathrm{I}, \mathrm{II})$, il faut distinguer le cas du mélange à $1 \%$ de néon des trois autres mélanges étudiés.

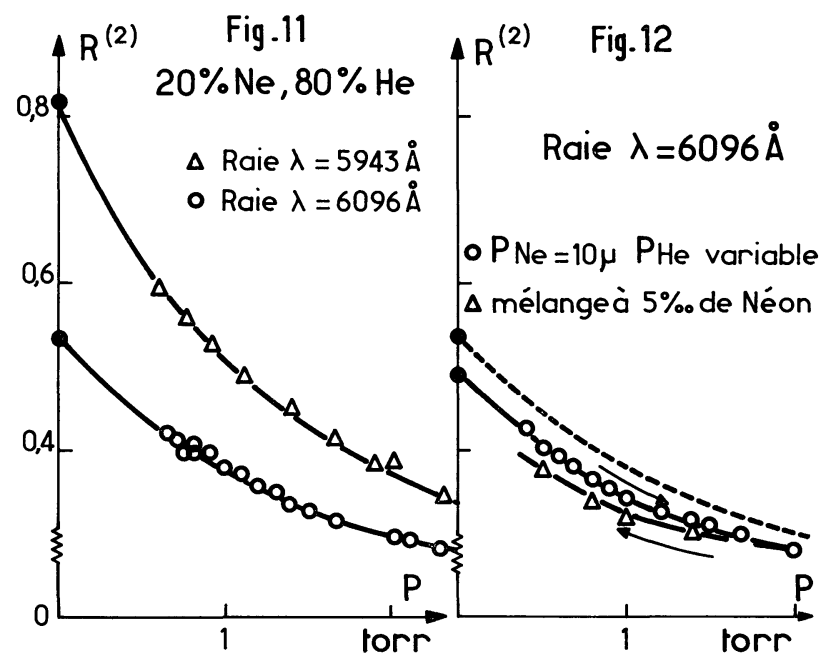

FIG. 11. - $R_{\mathrm{I}}^{(2)}$ et $R_{\mathrm{II}}^{(2)}$

pour un effet de cascade normal $(20 \% \mathrm{Ne}, 80 \% \mathrm{He})$.

FrG. 12.

$R_{\mathrm{I}}^{(2)}$ dans le cas de faibles pressions partielles de néon.

Examinons tout d'abord les résultats relatifs aux trois derniers. Les valeurs expérimentales de $R_{\mathrm{I}}^{(2)}$ et $R_{\mathrm{II}}^{(2)}$ pour le mélange à $20 \%$ de néon sont reportées en fonction de la pression, sur la figure 11, et les valeurs de $A^{(2)}$ et $F^{(2)}$ sur les figures 13 et 14 pour les trois mélanges.

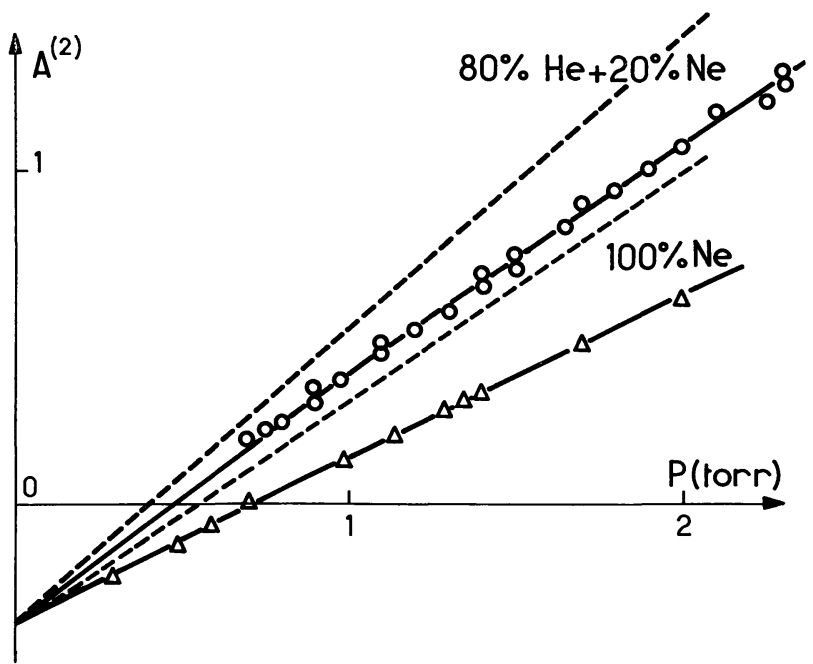

FIG. 13.

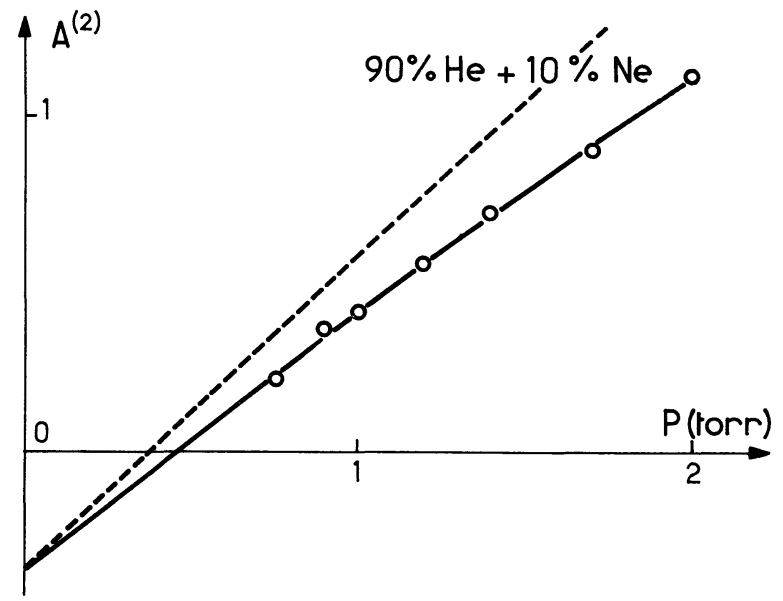

FIG. 13 a

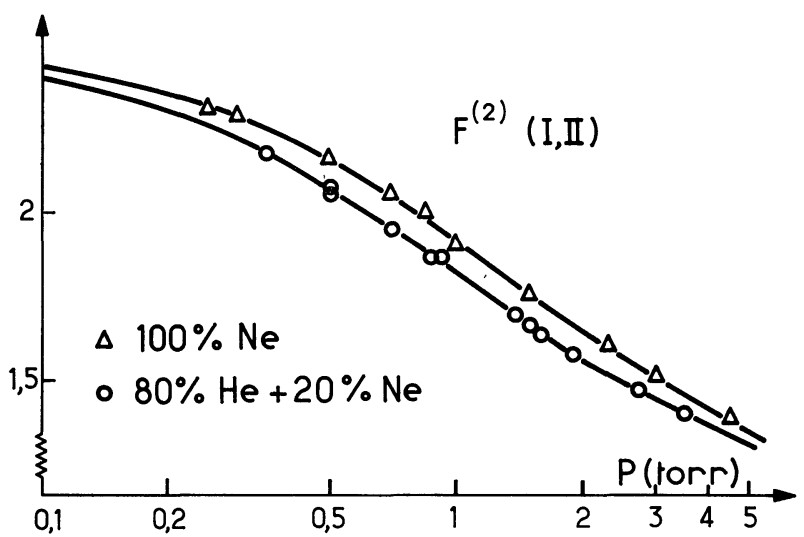

FIG. 14 .

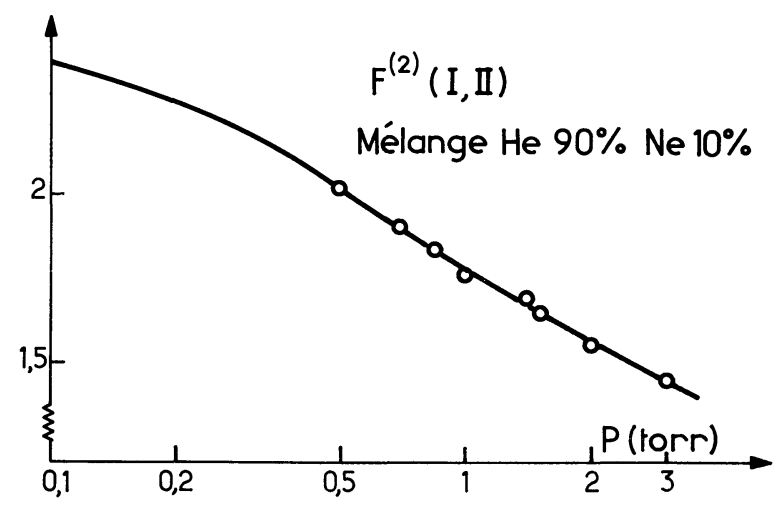

FIG. 14 a

Comme $\Gamma_{\mathrm{a}}(2)$ est connu à partir de l'étude des largeurs des courbes d'effet Hanle, $A^{(2)}$ et $F^{(2)}$ sont complètement déterminés par $\varepsilon_{0}$ et $\Gamma_{\mathrm{a}}(0)$. Aux pressions étudiées, $\Gamma_{\mathrm{a}}(0)$ est une fonction linéaire des pressions partielles de néon et d'hélium :

$$
\Gamma_{\mathrm{a}}(0)=\gamma_{\mathrm{a}}\left(1+\alpha_{\mathrm{a}} P_{\mathrm{Ne}}+\beta_{\mathrm{a}} P_{\mathrm{He}}\right) \text {. }
$$


Les trois paramètres $\varepsilon_{0}, \alpha_{\mathrm{a}}$ et $\beta_{\mathrm{a}}$ ont été ajustés de manière à coïncider au mieux avec l'ensemble des résultats des mesures de $A^{(2)}$ réalisées avec $20 \%$ et $100 \%$ de néon. Sur la figure 13, les courbes en trait plein sont les courbes théoriques (II.15), obtenues en posant :

$$
\varepsilon_{0}=0,20 ; \quad \alpha_{\mathrm{a}}=0,073 ; \quad \beta_{\mathrm{a}}=0,040 .
$$

Les courbes en pointillés correspondent à l'hypothèse $\alpha_{a}=\beta_{a}=0$ (pas de collisions de « quenching »).

Les valeurs des paramètres ainsi obtenues ont été utilisées pour construire la courbe $A^{(2)}(P)$ pour le mélange à $10 \%$ de néon ( fig. 13 a) et les courbes $F^{(2)}(P)($ II .13) pour les trois mélanges (fig. 14 et $14 \mathrm{a}$ ). L'accord excellent entre les points expérimentaux et les courbes théoriques est une bonne confirmation de la méthode de mesure et de la théorie (en particulier de l'existence d'un seul temps de relaxation pour l'alignement longitudinal et l'alignement transversal).

Nous pouvons donc écrire :

$$
\begin{aligned}
\Gamma_{\mathrm{a}}(0)=\gamma_{\mathrm{a}}[1+(0,040 & \pm 0,015) P_{\mathrm{He}} \\
& \left.+(0,073 \pm 0,030) P_{\mathrm{Ne}}\right]
\end{aligned}
$$

ce qui conduit aux sections efficaces :

$$
\begin{aligned}
& \sum_{\mathrm{Ne}-\mathrm{Ne}^{*}}(0)=(1,40 \pm 0,60) 10^{-15} \mathrm{~cm}^{2} \\
& \sum_{\mathrm{He}-\mathrm{Ne}^{*}}(0)=(0,52 \pm 0,20) 10^{-15} \mathrm{~cm}^{2} .
\end{aligned}
$$

Les sections efficaces $\Sigma(0)$ de destruction de la population (" quenching ») sont nettement plus faibles que les sections efficaces $\Sigma(2)$ de destruction de l'alignement.

Nous constatons d'autre part que le transfert de population par émission spontanée à partir du niveau $3 s_{2}$ est un effet important :

$$
\varepsilon_{0}=0,20 \pm 0,04 \text {. }
$$

Le transfert permet de comprendre les valeurs de l'anisotropie en champ nul observées aux faibles pressions. En effet, les valeurs expérimentales sont nettement supérieures aux valeurs théoriques obtenues si l'on ignore le transfert [2]. Toute mesure de section efficace par cette méthode est entachée d'erreur si l'on néglige $\varepsilon$.

En utilisant (II.11), la mesure de $\varepsilon_{0}$ permet de connaître la valeur de la probabilité de transition $\gamma_{\text {ba }}$ (donc la force d'oscillateur de la raie $3 s_{2}-2 p_{4}$ ) si l'on connaît $\gamma_{\mathrm{b}}$ et $\lambda$ par l'étude du niveau $3 s_{2}$. Ces mesures sont en cours.

c) Cas du mélange à $1 \%$ de néon. - Les valeurs de $R^{(2)}$ et de $A^{(2)}$ obtenues à partir du mélange à $1 \%$ de néon sont très différentes de celles que l'on calcule à partir des valeurs de $\varepsilon, \Gamma_{\mathrm{a}}(2)$ et $\Gamma_{\mathrm{a}}(0)$ déterminées ci-dessus. Pour les interpréter, il faut abandonner l'hypothèse de la réabsorption totale de la raie de résonance $3 s_{2}-1 p_{0}$.

L'étude directe du niveau $3 s_{2}$ avec ce mélange conduit par ailleurs à la même conclusion.
En effet, si la réabsorption de la raie de résonance n'est pas totale $(x<1)$, la correction de transfert :

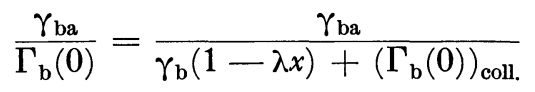

n'est plus égale au facteur $\varepsilon$ défini précédemment (II.25).

Ces expériences sont difficiles à interpréter pour ce mélange en proportions constantes. En effet, la pression partielle de néon variant avec la pression totale $P$, le coefficient de réabsorption $x$ de la raie de résonance varie et par suite la proportion de transfert par émission spontanée ne reste pas constante.

C'est la raison qui nous a conduits à réaliser une série d'expériences avec une pression partielle de néon constante et très faible $\left(P_{\mathrm{Ne}}=10 \mu\right)$ en augmentant progressivement la pression d'hélium. Les résultats sont reportés sur la figure 12. La courbe en pointillés correspond aux valeurs de $R$ calculées à partir de l'hypothèse $\gamma_{\mathrm{ba}} /\left(\Gamma_{\mathrm{b}}(0)\right)_{\text {rad. }}=\varepsilon_{0}=0,20$. Il est manifeste que cette hypothèse est à rejeter. Il y a par contre un bon accord entre les points expérimentaux et la courbe en traits pleins calculée avec l'hypothèse $\gamma_{\mathrm{ba}} / \gamma_{\mathrm{b}}(1-\lambda x)=0,13$.

$\mathrm{Si}$, après cette expérience, on pompe progressivement le mélange obtenu, on peut effectuer des mesures de $R$ à proportion de néon fixe $(5 \%$ dans le cas de la figure 12). On trouve des valeurs de $R$ sensiblement inférieures à celles obtenues à pression partielle de néon constante : dans la seconde expérience, la pression partielle de néon diminue; il en résulte une diminution du taux de réabsorption $x$ et, par suite, de l'importance de transfert. Dans les deux cas, les collisions $\mathrm{Ne}-\mathrm{Ne}^{*}$ sont négligeables : la variation de leur nombre ne peut donc expliquer la différence observée.

4. L'EFFet DE L'INTENSITÉ DE LA DÉGHARge. Revenons maintenant sur l'interprétation de l'effet de l'intensité $i_{\mathrm{c}}$ de la décharge excitatrice sur nos mesures.

Lorsque $i_{\mathrm{c}}$ augmente, on constate que la largeur $\Delta H^{(1)}$ des courbes d'effet Hanle diminue et que, d'autre part, $R_{\mathrm{I}}^{(1)}$ et $R_{\mathrm{II}}^{(1)}$ diminuent également. En d'autres termes, une augmentation de la décharge affine l'effet Hanle et simultanément dépolarise les raies de fluorescence. Ces deux constatations ne peuvent s'interpréter par des collisions. $\mathrm{Si}$ on admet, en effet, que la décharge augmente le nombre des collisions, on peut comprendre la dépolarisation observée, mais on s'attend alors à une augmentation de $\Delta H$ contrairement à ce qui est observé. Si en présence de la décharge se produit un effet thermique qui, au contraire, diminue le nombre des collisions, on interprète l'affinement, mais on ne peut plus comprendre la dépolarisation.

D'autre part, dans le néon pur, pour une pression de 0,3 torr et une intensité de décharge de $50 \mathrm{~mA}$, on a mesuré une valeur de $\Delta H^{(1)}$ inférieure à la largeur limite à pression nulle $\Delta H^{0}$, ce qui correspond à une 
durée de vie apparente supérieure à la durée de vie radiative de l'atome isolé.

Enfin, aucun affinement de ce type n'est observé sur le niveau $3 s_{2}$ dans le néon pur, alors que les modifications de largeur du $2 p_{4}$ sont très appréciables. En réalité, la précision est nettement moins bonne et on peut seulement affirmer qu'un affinement éventuel du niveau $3 s_{2}$ est au moins trois fois plus faible que celui du $2 p_{4}$ dans les mêmes conditions.

Cet ensemble de constatations nous a amenés à faire l'hypothèse de l'existence d'un phénomène de diffusion multiple cohérente lié à la présence du niveau métastable $1 s_{5}$ et des deux niveaux $1 s_{2}$ et $1 s_{4}$ très peuplés par suite du « trapping » des raies de résonance aux pressions de néon où nous opérons. Il est en effet prévisible que la population de ces niveaux et, par suite, le taux de réabsorption des raies de fluorescence issues de $2 p_{4}$ vers ces niveaux augmentent avec $i_{\mathrm{c}}$.

La théorie de la diffusion multiple permet une confrontation quantitative de l'affinement et de la dépolarisation observés.

En effet, d'après (I.12) :

$$
\begin{aligned}
& \Gamma_{\mathrm{a}}(2)=\left(\Gamma_{\mathrm{a}}(2)\right)_{\text {coll. }}+\sum_{\mathrm{i}} \gamma_{\mathrm{ai}}\left(1-x_{\mathrm{i}} \alpha(2, i)\right) \\
& \Gamma_{\mathrm{a}}(0)=\left(\Gamma_{\mathrm{a}}(0)\right)_{\text {coll. }}+\sum_{\mathrm{i}} \gamma_{\mathrm{ai}}\left(1-x_{\mathrm{i}}\right) .
\end{aligned}
$$

Il se trouve que dans le cas particulier des trois raies de fluorescence I, II et III, tous les $\alpha_{i}$ sont égaux à $\alpha=49 / 200$ [5]. Il est par suite intéressant de poser :

$$
x=\sum_{\mathrm{i}} x_{\mathrm{i}} \gamma_{\mathrm{ai}} / \gamma_{\mathrm{a}}
$$

$x$ représente la proportion de photons émis à partir du niveau $2 p_{4}$ et réabsorbés dans la cellule.

La variation de $x$ en fonction de $i_{\mathrm{c}}$ n'a pas de raison d'être simple. Pour cela, nous nous limiterons à l'étude de la variation des grandeurs observables pour les faibles valeurs de $i_{\mathrm{c}}$ (pente à l'origine). En utilisant (II.27) et les définitions (II.13) et (II.15), nous obtenons :

$$
\frac{\mathrm{d}\left(\Delta H^{(\mathbf{1})}\right)}{\mathrm{d} i_{\mathrm{c}}}=-\alpha \Delta H^{(\mathbf{0})} \frac{\mathrm{d} x}{\mathrm{~d} i_{\mathrm{c}}}
$$

$\frac{\mathrm{d} A^{(1)}}{\mathrm{d} i_{\mathrm{c}}}=\frac{40}{21}(1-\varepsilon) \frac{\gamma_{\mathrm{a}}}{\Gamma_{\mathrm{a}}(0)}\left[1-\alpha+\frac{\Gamma_{\mathrm{a}}(2)-\Gamma_{\mathrm{a}}(0)}{\Gamma_{\mathrm{a}}(0)}\right] \frac{\mathrm{d} x}{\mathrm{~d} i_{\mathrm{c}}}$

$\frac{\mathrm{d} F^{(1)}}{\mathrm{d} i_{\mathrm{c}}}=-\frac{280(1-\varepsilon) \gamma_{\mathrm{a}}\left[\Gamma_{\mathrm{a}}(2)-\alpha \Gamma_{\mathrm{a}}(0)\right]}{\left[20(1-\varepsilon) \Gamma_{\mathrm{a}}(2)-7 \Gamma_{\mathrm{a}}(0)\right]^{2}} \frac{\mathrm{d} x}{\mathrm{~d} i_{\mathrm{c}}}$.

Comme l'étude des collisions nous a permis de mesurer tous les paramètres figurant dans ces formules, la détermination expérimentale des variations de $\Delta H^{(\mathbf{1})}, A^{(\mathbf{1})}$ et $F^{(1)}$ constitue trois déterminations indépendantes de $\mathrm{d} x / \mathrm{d} i_{\mathrm{c}}$.

La variation de $F^{(1)}$ avec $i_{\mathrm{c}}$ étant en général très

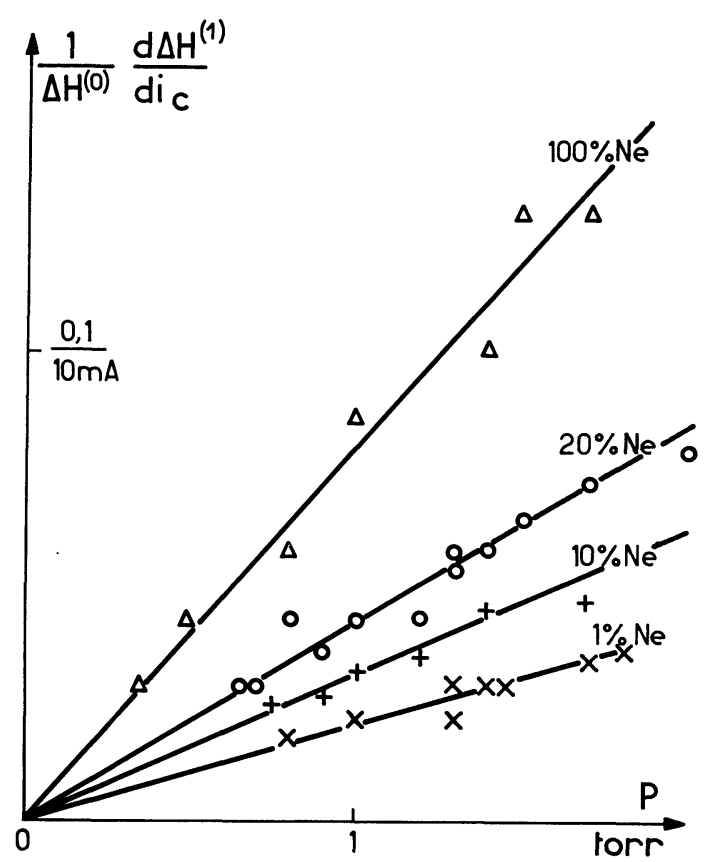

Fig. 15. - Affinement avec la décharge.

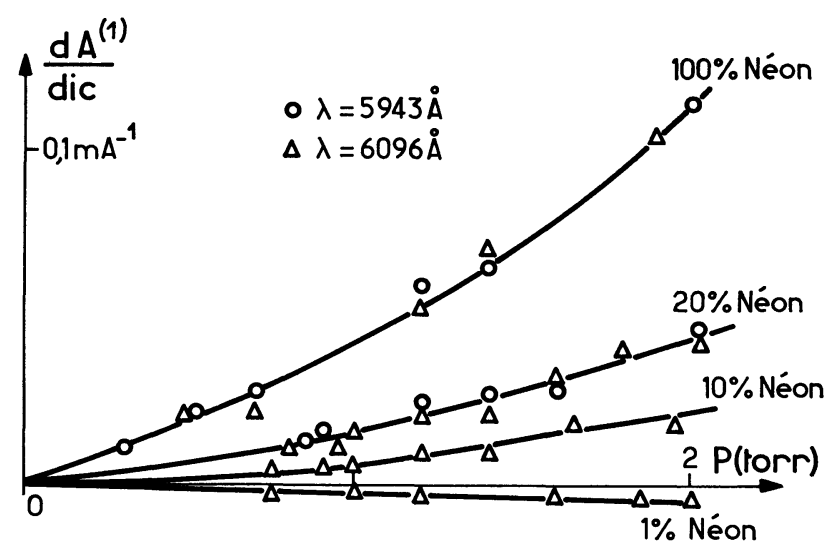

FIG. 16

faible, nous avons cherché uniquement à comparer les valeurs $\frac{\mathrm{d} x}{\mathrm{~d} i_{\mathrm{c}}}\left(\Delta H^{(1)}\right)$ déduites de l'affinement des courbes d'effet Hanle à celles que l'on obtient à partir de $A^{(\mathbf{1})}$, que nous appelons $\frac{\mathrm{d} x}{\mathrm{~d} i_{\mathrm{c}}}\left(A^{(\mathbf{1})}\right)$.

Les figures 15 et 16 montrent les variations de $\frac{\mathrm{d} \Delta H^{(\mathbf{1})}}{\mathrm{d} i_{\mathrm{c}}}$ et de $\frac{\mathrm{d} A^{(\mathbf{1})}}{\mathrm{d} i_{\mathrm{c}}}$ en fonction de la pression. Pour chaque proportion du mélange $\mathrm{He}-\mathrm{Ne}$, on en déduit que les valeurs de $\frac{\mathrm{d} x}{\mathrm{~d} i_{\mathrm{c}}}\left(A^{(\mathbf{1})}\right)$ et de $\frac{\mathrm{d} x}{\mathrm{~d} i_{\mathrm{c}}}\left(\Delta H^{(\mathbf{1})}\right)$ varient linéairement avec la pression (ceci apparaît sur la figure 15 pour $\frac{\mathrm{d} x}{\mathrm{~d} i_{\mathrm{c}}}\left(\Delta H^{(\mathbf{1})}\right)$ en raison de la proportion- 
nalité (II.29)), mais les deux déterminations sont différentes :

- Le rapport $\frac{\mathrm{d} x}{\mathrm{~d} i_{\mathrm{c}}}\left(\Delta H^{(\mathbf{1})}\right) / \frac{\mathrm{d} x}{\mathrm{~d} i_{\mathrm{c}}}\left(A^{(\mathbf{1})}\right)$ est de l'ordre de 1,3 pour le néon pur, de 1,8 pour le mélange à $20 \%$ de néon, de 2,5 pour le mélange à $10 \%$ de néon. Enfin, pour le mélange à $1 \%$ de néon, l'affinement reste détectable alors que l'on observe une augmentation très légère de la polarisation.

Il faut donc admettre que la diffusion multiple n'explique pas à elle seule les résultats observés. En particulier, les mesures faites pour le mélange à $1 \%$ de néon s'expliquent complètement en admettant que la décharge provoque un effet thermique qui diminue le nombre des collisions. La cellule est en effet reliée à un grand volume (verrerie, pièges...) et la décharge n'occupe qu'une faible partie de l'ensemble. Lorsque $i_{\mathrm{c}}$ croît, il est logique de prévoir une augmentation locale de la température $T$ au niveau de la décharge, alors que le reste de l'enceinte se maintient à la température ambiante. Dans un tel système, l'équilibre des pressions est assuré par une diminution locale de la densité de gaz. Comme, par contre, la vitesse moyenne croît, on peut prévoir une variation du nombre des collisions en $T^{-1 / 2}$.

Si l'on admet la diffusion multiple négligeable de ce cas, il est possible de mesurer avec précision, sur le mélange à $1 \%$ de néon, la modification relative du nombre des collisions provoquées, à chaque pression, par le courant de décharge. En admettant que cette modification relative est à peu près indépendante du pourcentage de néon (ce qui est très vraisemblable pour les mélanges à $20 \%$ et $10 \%$ de néon), on peut calculer la correction que cet effet thermique de décharge entraîne sur l'affinement et la dépolarisation.

Lorsque les valeurs expérimentales sont ainsi corrigées, les deux déterminations $\mathrm{de} \mathrm{d} x / \mathrm{d} i_{\mathrm{c}}$ coïncident à mieux de $20 \%$ près pour chacun des deux mélanges à $10 \%$ et $20 \%$ de néon ( fig. 17).

On constate dans ces deux cas que $\mathrm{d} x / \mathrm{d} i_{\mathrm{c}}$ est proportionnel à la pression partielle de néon (les pentes sont dans un rapport 2 ).

Remarquons enfin qu'en l'absence d'irradiation laser le rapport des intensités des raies de fluorescence I et II s'écrit :

$$
\frac{I_{\mathrm{I}}^{\pi}}{I_{\mathrm{II}}^{\pi}}=\frac{I_{\mathrm{I}}^{\pi}\left(i_{\mathrm{c}}=0\right)}{I_{\mathrm{II}}^{\pi}\left(i_{\mathrm{c}}=0\right)} \frac{1-x_{\mathrm{I}}}{1-x_{\mathrm{II}}} .
$$

Son étude expérimentale permet de séparer les contributions relatives des différentes raies. $\mathrm{Si}$ on fait l'hypothèse que seule la raie $\lambda=5943 \AA\left(2 p_{4}-1 s_{5}\right)$ est réabsorbée $\left(x_{\mathrm{I}}=x_{\mathrm{III}}=0 ; x_{\mathrm{II}}=x\right)$, on peut en tirer une dernière estimation de la quantité $\mathrm{d} x / \mathrm{d} i_{\mathrm{c}}$. Les points expérimentaux ainsi obtenus coïncident bien avec les deux autres mesures. On peut donc

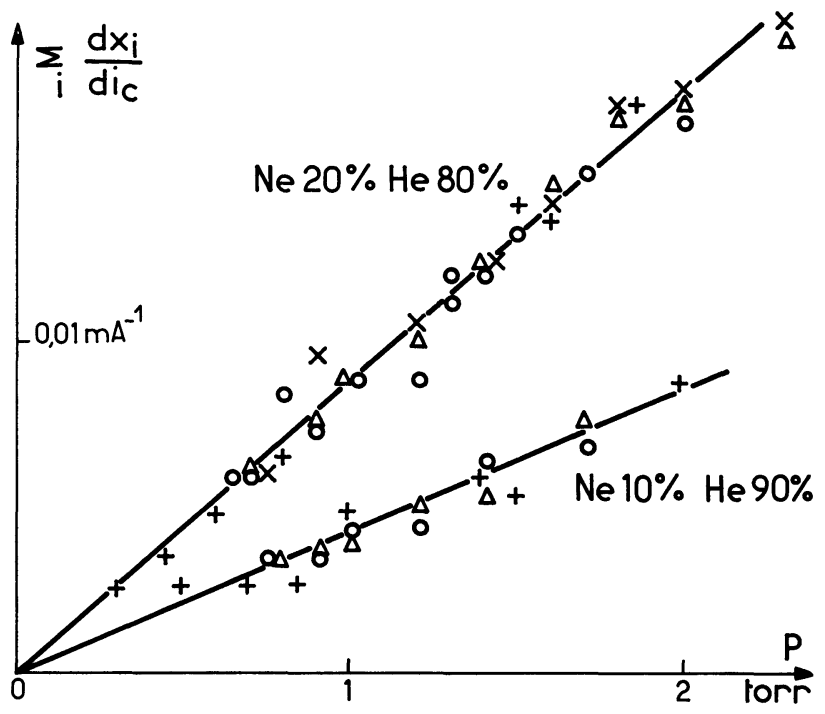

Fig. 17. - Différentes déterminations de $\mathrm{d} x / \mathrm{d} i_{\mathrm{c}}$ à partir de $\frac{\Delta I_{\pi}(\mathrm{I})}{\Delta I_{\pi}(\mathrm{II})}(+), \Delta H^{(1)}(\circ), A_{\mathrm{II}}^{(1)}(\Delta)$ et $A_{\mathrm{I}}^{(1)}(\times)$.

conclure que le phénomène de diffusion multiple cohérente se produit essentiellement sur la transition $2 p_{4}-1 s_{5}$. Ceci est facile à comprendre : le niveau $1 s_{5}$ est métastable et par suite beaucoup plus peuplé par la décharge que les deux niveaux $1 s_{2}$ et $1 s_{4}$ qui sont des niveaux de résonance.

L'accord entre les différentes déterminations pour le néon pur est beaucoup moins bon. Ceci n'est pas surprenant car les corrections de l'effet thermique sont beaucoup plus difficiles à évaluer dans ce cas. En effet, le comportement de la décharge étant fortement modifié par la présence de l'hélium, il n'est pas possible d'utiliser les résultats obtenus avec le mélange à $1 \%$. D'autre part, il semble bien que, dans ce cas, la diffusion multiple cohérente ne se produit plus uniquement sur la raie $5943 \AA$. Il convient enfin de souligner que nous n'avons pas introduit dans notre interprétation des phénomènes les collisions avec les électrons et les ions. A la précision actuelle de nos mesures, leur contribution n'est pas séparable de l'effet thermique dont nous venons de parler.

D. Conclusion. - Cette étude du niveau $2 p_{4}$ du néon nous a permis tout d'abord de vérifier l'ensemble des conclusions de l'analyse théorique : l'étude du rayonnement de fluorescence d'atomes excités irradiés par un laser permet de déterminer avec précision les relaxations des niveaux inférieurs ou supérieurs de la transition.

Pour obtenir des résultats significatifs, il est essentiel d'extrapoler toutes les mesures aux intensités de laser évanescentes. D'autre part, dans l'étude d'un niveau inférieur de la transition, il est indispensable de tenir compte du transfert par émission spontanée. 


\section{BIBLIOGRAPHIE}

[1] Parks (J. H.) et Javan (A.), Phys. Rev., 1965, 139, A 1351.

[2] Ḧ̈NSch (Th.) et Toschek (P.), Phys. Lett., 1966, 20, 273 ; Phys. Lett., 1966, 22, 150. Notre calcul tenant compte du transfert par émission spontanée nous permet de corriger la courbe théorique de la figure 4 de ce dernier article. Pour $P=0$, la valeur limite doit être 0,26 et non 0,338 . L'accord avec les points expérimentaux est alors bon.

[3] Decomps (B.) et Dumont (M.), C. R. Acad. Sc., 1966, 262 B, 1004.

[4] Decomps (B.) et Dumont (M.), C. R. Acad. Sc., 1966, $262 \mathrm{~B}, 1695$.

[5] Saloman (E. B.) et Happer (W.), Phys. Rev., 1966, 144, 7.
[6] Cette durée de vie est légèrement inférieure à la valeur $(18,5 \pm 1,5 \mathrm{~ns})$ mesurée par Bennett, qui n'a pas tenu compte de l'emprisonnement (" trapping ") de la raie $5943 \AA$ :

BENNETT (W. R.), KINDLMANN (P. J.) et MERCER (G. N.), Appl. Optique, Supplement on Chemical lasers, 1965, p. 34. KLOSE (J. Z.), Phys. Rev., 1966, 141,181 . La valeur trouvée par Klose (22 ns) est plus nettement différente.

[7] MiTcheis, (A. C.) et Zemansky (M. W.), Cambridge University Press, 1934.

[8] Piketity-Rives (C. A.), GrossetîtTe (F.) et BrosSEL (J.), C. R. Acad. Sc., 1964, 258, 1189.

Faroux (J. P.) et Brossei (J.), C. R. Acad. Sc., 1967, 264, 1452.

Barrat (J. P.), Casaita (Mlle D.), Cojan (J. L.) et Hamel (J.), J. Phys. Rad., 1966, 27, 608.

[9] Decomps (B.) et Dumon't (M.), à paraître. 